\title{
Selectively Targeting LTA4H Aminopeptidase Activity for the Development of Novel Anti-inflammatory Drugs
}

Schroeder M. Noble ${ }^{1}$, Kyung Hyeon Lee ${ }^{1}$, Elaine Cagnina ${ }^{2}$, Hoyoung Lee ${ }^{2}$, Marie Burdick $^{2}$, Y. Michael Shim ${ }^{2}$ and Mikell Paige ${ }^{3}$

${ }^{1}$ Department of Wound Infections, Bacterial Diseases Branch, Walter Reed Army

Institute of Research, 503 Robert Grant Avenue, Silver Spring, Maryland, USA, 20910.

${ }^{2}$ University of Virginia, Department of Medicine, Division of Pulmonary and

Critical Care Medicine. Charlottesville, VA

${ }^{3}$ Department of Chemistry and Biochemistry, George Mason University, Manassas, VA

Leukotriene $\mathrm{A}_{4}$ hydrolase (LTA4H) plays a critical role in inflammation, the immune response and host defense against infection. This bi-functional enzyme possesses epoxy hydrolase (EH) activity in inflammatory pathways and aminopeptidase (AP) activity in anti-inflammatory pathways. $\mathrm{LTA}_{4} \mathrm{H} \mathrm{EH}$ activity catalyzes hydrolysis of leukotriene $\mathrm{A}_{4}\left(\mathrm{LTA}_{4}\right)$ to $\mathrm{LTB}_{4}$, a proinflammatory lipid mediator that contributes to pulmonary inflammation, irritable bowel syndrome (IBS), COPD, and adult respiratory distress syndrome (ARDS). The LTA ${ }_{4} \mathrm{H} \mathrm{AP}$ activity catalyzes the hydrolysis of the peptide proline-glycine-proline (PGP), a chemotactic peptide resulting from breakdown of collagen. One of the hallmarks associated with inflammatory lung diseases is high concentrations of PGP, which maintains neutrophilic inflammation. Given its role in inflammation, several groups have developed compounds for the non-selective inhibition of $\mathrm{LTA}_{4} \mathrm{H}$ 's hydrolytic activity, which resulted in the simultaneous inhibition of both pro-inflammatory and anti-inflammatory pathways. Although the EH and AP functions of $\mathrm{LTA}_{4} \mathrm{H}$ share the same catalytic site, recently, we and others have sought to selectively target each function. We propose a novel therapeutic strategy of selectively augmenting $\mathrm{LTA}_{4} \mathrm{H} \mathrm{AP}$ activity with de novo preservation of the $\mathrm{EH}$ activity as treatment for inflammatory diseases. We have recently designed and tested a new molecule in two murine in vivo models to demonstrate potentiation of $\mathrm{LTA}_{4} \mathrm{H}$ AP activity as an effective therapeutic approach. This anti-inflammatory compound was evaluated for enhancement of $\mathrm{LTA}_{4} \mathrm{H}$ AP activity in kinetic assays, and the crystal structure of $\mathrm{LTA}_{4} \mathrm{H}$ bound to the compound was determined. The anti-inflammatory compound increased catalytic efficiency and substrate binding 10-fold. For structure determination, $\mathrm{LTA}_{4} \mathrm{H}$ crystal drops were overlaid with the antiinflammatory compound to achieve co-crystals that diffracted to $2.9 \AA$. The structure was determined using molecular replacement and revealed that the anti-inflammatory compound was stabilized by van der Waals interactions and hydrophobic interactions within the aromatic 
binding pocket of $\mathrm{LTA}_{4} \mathrm{H}$. Neutrophilic pulmonary inflammation and acute lung injury (ALI) were induced by intra-nasal lipopolysaccharide in the presence or absence of intra-nasal antiinflammatory treatment which selectively enhanced the $\mathrm{LTA}_{4} \mathrm{H}$ AP activity. This treatment protected murine lungs from ALI by significantly reducing lung edema and infiltration of neutrophils into the lungs. In conclusion, we have demonstrated enhancement of $\mathrm{LTA}_{4} \mathrm{H} \mathrm{AP}$ activity by an anti-inflammatory compound in vivo and in vitro, and determined the crystal structure of $\mathrm{LTA}_{4} \mathrm{H}$ bound to this compound. This structure will aid in the design of more potent small molecule compounds effective in potentiating LTA4H AP activity while preserving LTA4H EH activity. 